\title{
Transition from Water Resource System Modelling towards Integrated Water Modelling: Melbourne Water's Challenges and Experiences
}

\author{
$\underline{\text { M.D.U.P. Kularathna }}^{1}$, S. Ashbolt ${ }^{1}$, H. Jahanbani ${ }^{1}$, C.C. Daamen ${ }^{2}$, M. Mckerrow ${ }^{2}$ and B.G. Rhodes ${ }^{1}$ \\ ${ }^{1}$ Melbourne Water, Melbourne, Victoria, Australia, ${ }^{2}$ eWater Ltd, Bruce, ACT, Australia \\ Email: udaya.kularathna@melbournewater.com.au
}

\begin{abstract}
The management options available for balancing water supply and demand in metropolitan Melbourne have expanded significantly over the past two decades. The water supply system has transitioned from one that relied entirely on traditional surface water sources, multiple interconnected storage reservoirs and diversions structures, to one with a mix of water sources including decentralised supplies such as recycled water, storm water and household rainwater tanks, as well as a desalinated seawater supply. This range of 'alternative' water sources is expected to grow further in the future. Coincident to these infrastructure changes have been changes to water management arrangements: retail water companies, regional water authorities and the Victorian Environmental Water Holder are assigned water entitlements from the water supply system of which the assets are owned and operated by Melbourne Water. These water entitlements enable the above organisations to manage their water allocations independently. In its water storage operator role, Melbourne Water needs to ensure optimal management of the water supply system to meet the needs of the above water entitlement holders.
\end{abstract}

Water resource modelling tools are used at Melbourne Water to inform bulk water supply planning and management, drought response planning, water allocation, environmental flow planning and hydro-climatic impact assessment. In the past, water resource modelling had focused on the behaviour of the centralised bulk water supply system and therefore had limited ability to address new modelling needs. These include assessing: the water availability from centralised and decentralised supply sources, water allocation management options available to individual entitlement holders and the implications of local or centralised supply options on various components of the water cycle.

Melbourne Water, in consultation with its customers and stakeholders, has been developing a modelling tool, using eWater Source, to address the above needs. The comprehensive nature of this modelling tool has also highlighted the need for auxiliary modelling support tools. These include a documentation system for maintaining and sharing the knowledge base, a version control system to manage changes to model and data, a modelling workflow management system and a cloud computing system that enables faster processing of model runs. Development of modelling and auxiliary tools has been staged to ensure a smooth transition from the current modelling approach to the new system.

The paper will outline the historical progression of water resource modelling at Melbourne Water, drivers behind current modelling improvements, challenges faced in implementing new modelling capabilities, identified solutions and future directions.

Keywords: $\quad$ Water resource modelling, alternative water sources, water allocation, water cycle modelling 
Kularathna et al., Transition from Water Resource System Modelling towards Integrated Water Modelling...

\section{INTRODUCTION}

Managing the balance between water supply and water demand, particularly for large water supply systems that rely on natural water sources to service large populations, is a key challenge for water resource system managers. Modelling tools have assisted planners and managers to identify supply-demand solutions that provide for uncertainties in both future water availability and water demand. There are a variety of supplydemand-balance models used by practitioners and researchers. Sophistication and capabilities of such modelling tools have improved as new issues emerged in managing centralised water supply systems. These improvements have resulted in a shift from purely hydrologic models to those that include environmental considerations, economics and water quality aspects.

Melbourne Water is a Victorian government owned business that provides bulk water supply and wastewater services to Melbourne's retail water businesses and manages waterways and major drainage systems. Melbourne Water also supplies bulk water to several water authorities surrounding Melbourne. The water supply system managed by Melbourne Water is a complex interconnected system of 10 storage reservoirs, over 40 service reservoirs, 160,000 hectares of surface water catchments and a transfer system comprising hundreds of kilometres of pipelines, tunnels and aqueducts.

Water resource modelling tools are used at Melbourne Water to inform bulk water supply planning and management including decisions on water sourcing and system augmentation, drought response planning, water allocation, environmental flow planning and hydro-climatic impact assessment. The models also provide information to Melbourne Water's customers and stakeholders including: three retail water companies in Melbourne, five regional water authorities, Department of Environment, Land, Water and Planning, and the Victorian Environmental Water Holder. The models calculate the volumes of water that would be sourced, stored, released for the environment or transferred from various components of the water supply headworks system under various scenarios of climate, system management and water demand for up to several decades into the future. As with other large water businesses, Melbourne Water has been upgrading its water resource modelling tools in stages to meet various needs that emerged over time. Key past developments include the addition of environmental flow requirements and the optimisation modelling capability to identify management strategies that provide optimal trade-off of competing objectives.

The modelling tool capabilities are currently being enhanced, to address future needs identified by Melbourne Water through consultations with customers and stakeholders. These developments are undertaken using a model based on the eWater Source (Source) modelling platform (Carr and Podger, 2012; Welsh et al., 2012) to which Melbourne Water plans to transition from the currently used REALM suite of modelling tools (Perera et al., 2005). The enhanced model development program includes both enhancements to Melbourne Water's Source based headworks model as well as to the Source modelling platform itself. The key activities of the enhanced headworks model development program include consultation with customers and stakeholders, developing new modelling capabilities, calibration and testing of the new model to assess its suitability to replace the existing modelling tool in supporting various key decisions processes, developing supplementary computer tools including the setting up of knowledge management, version control, model run processing and workflow management systems.

This paper is structured as follows. Section 2 describes the drivers behind developing the new capabilities and transitioning to the new modelling platform. Section 3 outlines the challenges in implementing the new capabilities and their solutions. Section 4 outlines future directions for modelling capability development. Section 5 presents the conclusions and recommendations.

\section{DRIVERS FOR MODELLING IMPROVEMENTS}

Melbourne Water's transition from traditional water resource modelling to integrated water system modelling is in response to several drivers. They are:

(1) The changing mix of water supply sources available in Melbourne: Future supply demand assessments need to represent the growing number of decentralised supply sources available in Melbourne including rainwater tanks, recycled water schemes and storm water reuse as well as the interactions between different water cycle components.

(2) Water entitlement framework in Melbourne that assigns water entitlements to the Victorian Environmental Water Holder (VEWH) and the primary entitlemnt holders (PEHS) comprising retail water companies and the regional water authorities: Accounts of individual PEHs hold all water that would be available for urban consumption during the current year under a very dry scenario. Individual 
Kularathna et al., Transition from Water Resource System Modelling towards Integrated Water Modelling...

entitlement holders manage their share of water through specific water sourcing strategies. The capability to model individual water accounts and assess differential management actions is needed to identify options that provide optimum outcomes for the overall system.

(3) The need for improved transparency and knowledge management: Water resource modelling informs stakeholders and customers on a range of water management decision areas that call for improved transparency. The increasing complexity of modelling tools intensifies this need. Parallel to this is the need to retain and manage modelling knowledge associated with a multifaceted model. This need is difficult to be met by the past approaches of model custodians and standard linear documentations.

(4) The need to maintain one modelling tool for a range of purposes: The limitations of the current modelling platform to provide for different purposes necessitated multiple models in the past. These included a model for simulation modelling (with two separate versions: a monthly time step model for long term assessments and a daily time-step model for more detailed short-term assessment), a model for optimisation modelling and a model that computes water allocations. Multiple versions of the model caused difficulties in model maintenance and inefficiencies in the provision of modelling information.

(5) The need for fast turnaround of modelling outcomes: Water Resource Modelling, in particular, those that aim to model interactions of water cycle components or identify optimal water resource management solutions are computationally intensive. Internally maintaining computer hardware to address such needs has been impractical in the past, which required the modelling problem to be simplified to one that can be solved with limited computational capability. The advances in cloud computing facilities provide an opportunity to access virtually unlimited computing power at gradually reducing costs.

(6) The need for a smooth transition to a next generation modelling platform: To address the above needs, it is necessary to transition to the Source platform that provides the flexibility and extensibility to address the above needs. As the current suite of modelling tools is constantly being used and updated, the transition process needs to be undertaken in several stages to provide a smooth transition.

\section{CHALLENGES AND SOLUTIONS}

This section describes the challenges posed by each of the improvement drivers outlined in Section 2 and the solutions identified to address the challenges.

\subsection{Representing the Changing Mix of Supply Sources}

The capacity to calculate the water availability and demand within a single model provides the opportunity to explore optimisation of supply demand balance for efficiency and cost. Stakeholders were interested in investigating the changes in potable water demands on the centralised water supply system that result from integrated water management solutions by water retailers, primarily through sourcing local water supply from alternative sources.

This integration was a major step in the model representation which previously focused on the water sources available from the centralised water supply system and relied on demand estimates developed externally. The water supply system has a large storage capacity of about $1812 \mathrm{GL}$ in comparison to the annual urban water demand and the annual average inflow both of which are about 420 GL. The water held in storage reservoirs provide a large buffer against fluctuations in water demand and inflow over not only daily and monthly time frames but also over multiple years of up to 15-20 years into the future. A monthly time step model can adequately represent these characteristics of the centralised system for long-term planning purposes. In contrast, the water available from the decentralised sources (e.g. rain water tanks) provides a much smaller and short-term buffer against fluctuations in inflow and demand. An adequate representation of the use of these sources requires a more granular representation of water demand, requiring the use of a daily or subdaily time step. One key challenge posed by this was in accommodating, within one model, the differences in granularity required in modelling the centralised and decentralised systems as shown in Figure 1.

Data available for modelling decentralised sources and demands was the second challenge. As an example, Melbourne Water or retail water companies are not able to accurately assess the number of rain water tanks in a specific local area and how they are being used. Data availability is also affected by the frequency of water meter readings (currently 3 -monthly). 
Despite these general limitations in data availability, Melbourne's retail water companies require a more detailed assessment of the water demand than that required by Melbourne Water as the bulk water supplier. This posed the third challenge which is to develop a modelling methodology that is scaleable for use with a different level of granularity by different water companies.

Solution to Representing the Changing Mix of Supply Sources: The modelling tool Urban Developer (Hardy et al., 2011) was used as the foundation of the implementation of a local demand/supply model within Source. A 'template' approach is used to allow representation of urban water use at a scale appropriate for demand model configuration. This is then aggregated within the headworks model to represent demand at a more appropriate sub-regional scale. Each template, comprising a series of urban specific water-use nodes, can be configured to represent a specific mix of urban demands. These templates can in turn be combined in different proportions to model the water uses in different areas. De-centralised alternative sources are also represented at this level and interact with the demands generated by the template to produce a net mains supply requirement. In the case study implemented, four different dwelling types: unit, townhouse, free-standing, and free standing with rainwater tanks were considered sufficient to represent the modelled area.

Urban water user nodes within urban templates are underpinned by a behavioural model that provides the simulation of water-use using the Behavioural End-use Stochastic simulator (BESS) of Thyer et al. (2011). BESS stochastically simulates various end-uses (outdoor, shower, washing machine, toilet, tap, etc.) at the household scale at sub-daily time steps.

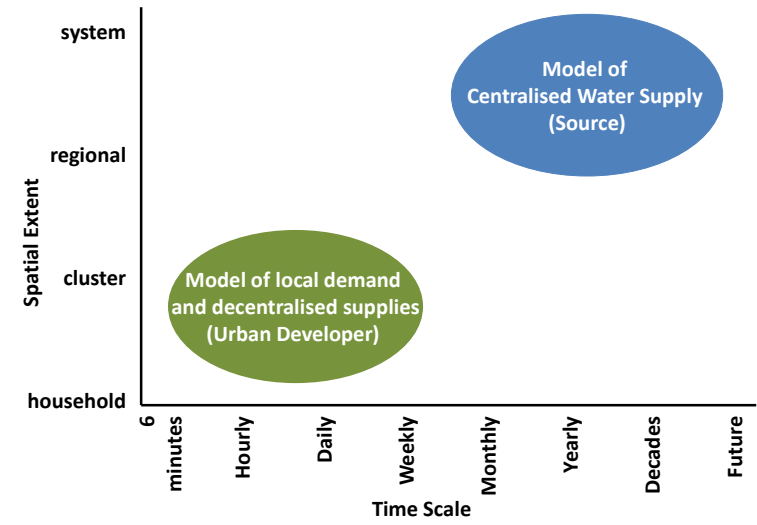

Figure 1. Spatial and Temporal Extents Represented by models

\subsection{Representing the Water Entitlement Framework}

Capability to assess and optimise the management of water allocations from the centralised system by seven PEHs and the VEWH required development. Melbourne Water as the bulk water supplier is responsible for undertaking the modelling to calculate the water allocations and update water accounts of each PEH at the beginning of every month. The likely volume of water available for urban consumption over the water year (from 1 July to 30 June) determines the water allocation, after allowing for various commitments. It is calculated based on the volume in storage and future inflow, after allowing for the dead storage, water already held in specific accounts of PEHs and VEWH, future volumes needed for meeting passing flow requirements, unharvestable future inflow and future system losses. Use of the lowest recorded inflow conditions for the rest of the year is essential in this approach. The resulting conservative estimate increases from one month to the next as water flows into the system (assuming that next month's actual streamflow would be higher than the lowest recorded streamflow volume that is used in the allocation estimation). By definition, the above calculation is forward-looking. Forward looking calculations pose a challenge in representing water allocation within a simulation model in which the system behaviour is modelled using a sequence of discrete time steps, one time-step (month) at a time without a forward-look.

The model also needs to represent a set of detailed rules defined by Melbourne's water allocation framework. Even though some of these rules are similar to those used in other river basins within Australia, developing the modelling capability to represent these rules, while providing the flexibility to include possible future amendments was another modelling challenge.

Management of environmental and urban water allocations is significantly different. Environmental flow requirements are dynamic across multiple time scales. The representation of environmental flow requirements requires the creation of rules to replicate complex patterns. They include the frequency of release, volumes needed to achieve desired river levels, the order of priority for meeting the flow requirements at various locations, and the need use the environmental allocation optimally by creating high flow events through 'piggy-backing' on naturally occurring high flow conditions. The challenge is to configure Source to reflect the diverse nature of ecological system requirements and the impact on the environmental water holdings and the rest of the system. 
Solution to Representing the Water Entitlement Framework: The forward-look required within the model for water allocation assessment for PEHs was incorporated by including a sub-model (a Source 'plug-in') that runs an identical second Source model ('Allocation Model') at the beginning of each timestep of the main model. The main model simulates the future scenario. At each time step, the sub-model is run to provide an estimate of water allocations assuming a dry scenario. At each call from the main model, the sub model simulates the Dry (worst case) scenario until the end of the water year to provide a conservative estimate of allocation. Even though this approach had been previously implemented by Melbourne Water using the REALM platform, its capabilities were limited to assessing the water allocations. The implementation on Source provides the flexibility for assessing various management actions by the PEHs such as sourcing additional water supplies or demand management.

The capability to represent the management of environmental water allocation is being developed using two main mechanisms, the Environmental Flow Node (EFN) and the Environmental Flow Manager (EFM). The Environmental Flow Node is used to generate 'order volumes' to meet instream environmental requirements at an individual asset. One or more configured actions define the desired flow patterns including start criteria, desired flow response and frequency, as well as criteria for success of the action and condition of the asset targeted by the action. The new Environmental Flow Manager will prioritise environmental flow actions defined at Environmental Flow Nodes throughout the system. The EFM will also handle management of accounts, and apportionment of water from the accounts to particular environmental events. These two network elements interact with each other and the rest of the network, including the Resource Assessment System, to represent the management of environmental water. This approach will provide the capability to identify optimal management strategies under future climatic scenarios. Melbourne Water is contributing to a dedicated technical group led by the Murray-Darling Basin Authority that is implementing the above enhanced representation of environmental flow in Source.

\subsection{The need for Improved Transparency and Knowledge Management}

Transparency of water resource decisions has always been an important consideration for Melbourne Water, Melbourne's retail water companies and their stakeholders. There has been a continual increase in the range of questions answered (and decisions informed) by water resources modelling on hydrological, climate change, environmental, economical, and regulatory aspects. Future questions will include those on integrated water supply assessment taking into account various components of the urban water cycle. In developing the capability to answer the above questions, the modelling tools and datasets have become increasingly more complex. The modelling knowledge base has grown to a level that the traditional approaches to relying on a limited number of modelling experts and linear documentations are unsuitable in managing the knowledge base and improved transparency. These complexities highlighted the need for two new capabilities. The first is the need for an automated capability within the modelling system to reveal the assumptions that have been used in undertaking particular modelling assessments or developments over a period. The second is a suitable knowledge management system that is easy to refer to, update and share.

Solution to the Need for Improved Transparency and Knowledge Management: A combination of autogenerated reporting tools and online manual documentation was implemented to ensure model information was given adequate context to be useful for a range of purposes. The improved audit log feature within Source keeps track of the changes made to a modelling project over time. Online, wiki-style documentation was developed from existing modelling documentation into a form that is easy to update, accessible, and provides the ability to control sections to be public, stakeholder view, or internal view only.

\subsection{The Need to Maintain One Modelling Tool for a Range of Purposes}

In the past, the expansion of services provided by the water resource model could not be met by a single model. Instead, multiple models were created to address different needs. Expansion resulted in three models with corresponding increases in the efforts required to maintain the model. The maintenance of these models involved various continuous improvements as well as configuring them to reflect the current operating and asset management arrangements and plans. Even though the data inputs used in these models had the same basis, there were differences that required tailoring the inputs to suit each model. The pre-processing and post-processing tools used for analysing the outputs of these models were in-turn different and required regular updates. The result of the above was that not all of the models were able to be kept up-to-date at all times. Instead, they were updated when needed, causing delays in providing modelling solutions and increasing the possibility of inconsistencies in modelling. The above issues highlighted the need to maintain one model for all water resource modelling purposes including long-term water resource planning, environmental flow planning, operating strategy optimisation and water allocation. The main challenge posed 
by this was in developing the flexibility within the modelling platform to switch easily between different modes or purposes and switching various assessment components on or off depending on the selected purpose to improve model performance.

Even with the creation of one model as the base model, ongoing development and assessments will result in a hierarchy of models, with parent model as the root and subsequent child models that will, in turn, have their child models as well. A system to assist managing the hierarchical model structure and associated datasets to enable tracing of their development trails was needed. In addition, potential improvements to models and datasets are frequently identified. The improvements are implemented based on a consideration of their priorities. The need to streamline the management of model improvement workflow has become a new challenge as the modelling system and data sets become increasingly complex.

Solutions to the Need to Maintain One Modelling Tool for a Range of Purposes: The model was developed with the flexibility to run on either a monthly timestep or a daily timestep. This made the model suitable for both long-term water resource assessments that require monthly timesteps as well as more detailed or shortterm assessments that require daily timesteps. A Source platform feature - Scenario Input Sets - was used to switch between input data sets with monthly timesteps and those with daily timesteps. Functions used within the model were adapted to allow for differing timesteps on the basis of selection of a particular scenario input set. As described previously, the same model was configured for the modelling of water allocation management using monthly time steps. In addition to the above purposes, the model is planned to be expanded to include the constructs required for optimisation modelling studies as well. This involves creating functions within the Source model to define objective function(s), decision variables and constraints that describe the optimisation problem. To reduce model run times, it is possible to turn off complex calculations that are not required for the assessment being undertaken.

A model repository system is being developed to track changes to the model (including updating of parameters and inputs) and the effects on outputs in addition to tracking the effects of developments of the software platform. A shared workflow management system has been setup enabling the model users to record model improvement needs and their priorities and assign the tasks to appropriate personnel.

\subsection{The Need for Fast Turnaround of Modelling Outcomes}

Model run processing of the water resource model for some purposes requires long computation times. Computational times are particularly significant for modelling that involves a large number of stochastically generated scenarios, or those that aim to optimise management strategies that provide long-term benefits. With Melbourne Water's models, the former can take several hours whereas the latter can take several days or weeks when undertaken using an optimisation algorithm such as Genetic Algorithms that Melbourne Water currently uses for assessing optimal system management strategies. The turnaround time of modelling outcomes becomes critical when responding to informing key decisions within tight deadlines. Long turnaround times create inefficiencies in modelling improvements and assessments that require experimentation. The challenge in this case was to develop an extensible cloud computing portal and process that provide modellers with direct access to cloud resources.

Solution to the Need for Fast Turnaround of Modelling Outcomes: The option of running the Source model on the Cloud has now been implemented as a pilot and allows large numbers of replicate model runs and complex optimisation runs to be completed quickly and efficiently. It facilitates access to a large number of virtual machines with the ability to select a particular price model. This provides a resource that has maximum flexibility to deliver results within required time and cost constraints.

\subsection{The Need for a Smooth Transition from a Fully Operational Modelling Platform}

The previous water resource planning model informed many decision processes and therefore has been extensively reviewed and tested. It was important to demonstrate consistency in old and new model outcomes with equivalent performance for all applications. The transition period necessitated maintenance of both old and new models. This was a challenge because the new model is being developed fast, and the old model is continually being updated to include various assessment requirements. The need to develop internal skills while major developments of the new model were being undertaken externally, was also a key consideration.

Solution to the Need for a Smooth Transition from a Fully Operational Modelling Platform: Transition to the new platform is being undertaken through a three stage process. The first stage involves calibration of the new model against a compatible version of the old model. Both modelling tools will then be used in parallel 
Kularathna et al., Transition from Water Resource System Modelling towards Integrated Water Modelling...

for various applications to compare their performances. A final calibration against past operation of the system is planned on completion of the new model development. Transition activities are undertaken internally, in close cooperation with external developers, to facilitate internal skill development. The development project is delivered using agile approach to enable new capabilities to be incrementally tested.

\section{FUTURE DIRECTIONS}

Future assessments of water supply and demand balance will require further improvements to the modelling of interactions between water cycle components. These include the use of storm water and its implications on urban waterways as well as wastewater reuse. While the Source modelling platform has the core capabilities to address these, further investigations are planned in collaboration with Melbourne Water's customers and stakeholders to identify future development needs. A modelling information system is planned to be developed to initially provide the customers and stakeholders (retail and regional water companies, DELWP and VEWH) with the ability to access information from modelling assessments undertaken by Melbourne Water. The system is planned to be expanded to provide direct access to modelling tool and facilitate linkages to customers' modelling systems. The cloud computing system developed in the current project will provide the platform for achieving this goal. It also has the potential to facilitate joint assessment of water availability from interconnected water supply systems surrounding Melbourne.

\section{CONCLUSIONS AND RECOMMENDATIONS}

Water resources modelling at Melbourne Water has been advancing in stages in the past to address the needs of customers and stakeholders. The modelling has become increasingly complex over the years, with a corresponding expansion of the role of modellers. A new modelling system is currently being developed based on Source modelling platform to cover multiple disciplines. They include hydrology, water demand estimation, economics, system optimisation and regulatory aspects. The system is capable of representing the growing mix of water sources available in Melbourne. Further expansion of the modelling system is expected in the future to enable better understanding of the water cycle management options. The development, maintenance and use of complex models have posed many challenges in the past. The past solutions to these challenges relied heavily on the expertise and skills of modellers. With the growing complexity of the models that cover multiple disciplines, the past practise is becoming increasingly insufficient. Present day modelling requires the support of auxiliary systems for many reasons. They include the need for knowledge management, succession planning, modelling workflow management, model/data version control, model run processing, sharing information with customers and stakeholders and improved transparency.

Streamlining of modelling systems is essential, as has been experienced by Melbourne Water. Multiple modelling systems cause significantly difficulties and inefficiencies. Changeover of modelling systems that are being used to inform many decisions takes time and significant effort. Hence the modelling systems need to be extensible and flexible to ensure they are "future proof" over their expected lifetime.

When the modelling systems approach their end-of-life, development of new modelling systems requires specialist skills and expertise including software development that may need to be outsourced. This poses the challenges of knowledge sharing with the external developers, as well as the need to maintain internal skill development. With many new developments, the software solutions need to evolve as alternative solutions are investigated, tested and refined. These developments are best delivered incrementally through agile delivery processes that are flexible in regards to the project scope. Finally the transitioning to a new modelling system needs to be well planned to ensure that the new system provides consistent or better information while the existing system is still being used to inform decisions.

\section{REFERENCES}

Carr, R., Podger, G. (2012). eWater Source - Australia's Next Generation IWRM Modelling Platform, 34th Hydrology and Water Resources Symposium, ISBN 978-1-922107-62-6.

Hardy, Matthew J., McArthur, Jane C., and Hardy, Mathew J. (2011). Urban Developer Technical Overview, eWater Cooperative Research Centre, Canberra.

Perera, B.J.C., James, B., and Kularathna, M.D.U. (2005). Computer software tool REALM for sustainable water allocation and management. Journal of Environmental Management, Elsevier, 77, 291-300.

Thyer, M., Micevski, T., Kuczera, G., and Coombes, P. (2011). A Behavioural Approach to Stochastic End Use Modelling. Oz Water, 9-11, Adelaide.

Welsh, W.D., Vaze J., Dutta, D., Rassam, D., Rahman, J.M., Jolly, I.D., Wallbrink, P., Podger, G.M., Bethune, M., Hardy, M., Teng, J., Lerat, J. (2012) An integrated modelling framework for regulated river systems. Environmental Modelling and Software, DOI 10.1016/j.envsoft.2012.02.022. 